\title{
V2, PHASES, ET PÉRIPHÉRIE GAUCHE : REMARQUES SUR LA TOPICALISATION EN FRANÇAIS ET EN NORVÉGIEN
}

\author{
ESPEN KLÆVIK-PETTERSEN \\ Université d'Agder
}

RÉ S U MÉ

\begin{abstract}
Dans les langues romanes et germaniques, le procédé de topicalisation consiste à déplacer en début de phrase un constituent pour lui conférér une interprétation précise, à savoir celle d'un topique/thème. Au-delà de cette caracteristique partagée, les deux groupes de langues se distinguent par deux aspects purement formels : les langues romanes ont recours à un élément de reprise sans autre changement dans l'ordre des mots, tandis que les langues germaniques V2, de façon inverse, emploient l'inversion sujet-verbe sans élément de reprise. Dans cet article, je compare la topicalisation en français et norvégien standards. En m'appuiant sur la théorie des phases (Chomsky, 2000), je propose que le type de topicalisation (avec vs. sans reprise) et le mouvement du verbe ( $\mathrm{T}^{0}$ vs. $\left.\mathrm{C}^{0}\right)$ sont étroitement liés. L'hypothèse présentée est que la périphérie gauche d'une langue V2 et celle d'une langue non V2 sont différentes, et que le facteur décisif est le statut phasal ou non de la tête Fin ${ }^{0}$.
\end{abstract}

Mots-clés : topicalisation, périphérie gauche, théorie des phases, V2

\section{[1] INTRODUCTION}

Si les langues romanes et les langues germaniques possèdent toutes un procédé de topicalisation consistant à déplacer un constituant en début de phrase, il est généralement admis que les deux groupes de langues ne procèdent pas de façon identique à cet égard. ${ }^{1}$ Dans un article récent, Helland et al. (2020) discutent des différences subtiles entre le français standard et le norvégien en ce qui concerne la topicalisation. Les auteurs proposent que les différences observées sont le reflet d'une organisation différente de la périphérie gauche dans les langues concernées.

C'est dans la continuité de ce travail que la présente contribution cherche à développer encore l'hypothèse d'une distinction syntaxique fondamentale entre le français et le norvégien en proposant une analyse de la topicalisation basée sur

[1] Je souhaite remercier un relecteur anonyme pour ses remarques constructives sur une version antérieure de cet article. 
la théorie des phases (Chomsky 2000 et seq.) Reliant la topicalisation et le mouvement $\mathrm{du}$ verbe fini, je me propose de formuler une hypothèse nouvelle sur la syntaxe propositionelle des langues $\mathrm{V}_{2}^{2}$ et non-V2 qui fournit une définition précise de la périphérie gauche basée sur la notion de phase et non pas sur le domaine syntaxique (le CP). Je soutiendrai que les deux (groupes de) langues se distinguent par le fait que la topicalisation est une dislocation en français et non pas en norvégien et que la dérivation de cette construction n'implique la périphérie gauche qu'en français.

La structure de l'article est la suivante : d'abord je présenterai brièvement la construction à l'étude et les différences pertinentes entre le français et le norvégien à cet égard. Dans la section [2] seront introduits les outils théoriques qui vont servir de base à l'analyse de la topicalisation, à savoir le modèle cartographique de la périphérie gauche ainsi que la théorie des phases. La section [3] présente une analyse de la topicalisation en français et en norvégien. Ensuite seront présentées quelques données tirées de l'ancien français dans le but de démontrer que la distinction pertinente est bien celle entre les langues V2 et non V2, et non pas entre les langues germaniques et les langues romanes.

\section{[1.1] La topicalisation en français et en norvégien}

Il convient de clarifier d'emblée quelle construction est en jeu dans cet article, car le terme topicalisation est parfois pris dans un sens large qui déborde l'acception que je lui donne ici. Par ce terme, j'entends le procédé par lequel un groupe nominal ou pronominal se trouve déplacé en tête de phrase et reçoit une interprétation comme le thème ou topique de la proposition. Cela pourrait en principe s'appliquer même au sujet, qui se trouve en première position de la phrase en français comme en norvégien et auquel on associe très souvent une interprétation thématique. Sans prétendre que le sujet ne puisse être un topique, je souhaite toutefois exclure le cas non marqué où le sujet débute la phrase, car ce qui nous intéresse dans cet article est surtout la transformation opérée sur la proposition de base qui déplace un constituant en tête de phrase. ${ }^{3}$ Seront donc seulement considérés les topiques non-sujets.

Les langues romanes et germaniques se distinguent nettement par rapport au

[2] Le relecteur demande quelle est la définition d'une langue V2. Si plusieurs sont possibles, aucune n'est complètement satisfaisante ; pour une discussion, voir Klævik-Pettersen (2019: 69-72). Dans le cadre de cet article, il suffit de considérer qu'une langue $\mathrm{V} 2$ requiert le mouvement du verbe fini au domaine $\mathrm{CP}$.

[3] Je dois encore souligner que cette transformation peut bien s'appliquer au sujet, mais vu que cela ne produit aucun changement dans l'ordre des mots, ni en français ni en norvégien, il est difficile de prouver avec certitude que l'on est en présence d'une topicalisation dans ce sens du mot. Selon certains linguistes (voir par exemple De Cat 2007), la topicalisation du sujet équivaut à une dislocation en français, entrainnant donc toujours un pronom de reprise ; mais cela ne veut pas forcément dire qu'un sujet in situ ne puisse être interprété comme le thème de la proposition. En norvégien et les langues scandinaves en général, l'absence d'indices morphosyntaxiques rend très ardue la tâche de distinguer entre un sujet in situ et un sujet topicalisé. 
procédé de topicalisation. À titre d'illustration, considérez les exemples suivants tirés du français et du norvégien standards. En français, la topicalisation d'un constitutant (en l'espèce le complément d'objet direct) à la périphérie gauche requiert forcément l'emploi d'un pronom clitique de reprise dans la proposition, mais elle ne déclenche aucun autre changement dans l'ordre des mots (1). Le norvégien, par contre, procède de façon inverse: la topicalisation entraîne l'inversion $\mathrm{du}$ sujet et du verbe fini, conformément à la règle $\mathrm{V} 2$ en vigueur dans les langues germaniques (sauf l'anglais), mais l'emploi d'un pronom de reprise est hors de question (2):

(1) Ce livre, Hans Petter l'a écrit pour ses étudiants

(2) Denne boken har Hans Petter skrevet for studentene sine

$\mathrm{Ce}$ livre a Hans Petter écrit pour étudiants.DEF ses

Cependant, bien qu'il soit courant d'associer le statut V2 des langues germaniques et la topicalisation sans reprise, il n'est à priori pas clair qu'il existe de lien logiquement nécessaire entres ces deux propriétés. Autrement dit, il nous faut expliquer pourquoi le français n'accepte pas la topicalisation sans reprise (3), et pourquoi le norvégien n'accepte pas la pronominalisation avec reprise (4) : ${ }^{4}$

(4) *Denne boken har Hans Petter skrevet den for studentene sine

À première vue, il pourrait sembler que les questions soulevées soient d'un assez faible intêret. Après tout, dirait-on, il existe un consensus général pour dire que les topicalisations en français sont des dislocations (Blasco-Dulbecco, 1999; De Cat, 2007; Helland et al., 2020) et que l'emploi du pronom de reprise découle naturellement de ce constat. ${ }^{5}$ Or, cette réponse ne fait que repousser la question : pourquoi la topicalisation en français implique-t-elle une dislocation, tandis que cela n'est

[4] Comme le fait remarquer le relecteur, (3) est marginalement acceptable en français si le premier constituent est interprété comme un focus (auquel cas il faudrait plutôt écrire : 'CE LIVRE, Hans Petter ...'). Le focus initial a un contour prosodique et une interprétation différents, évoquant le plus souvent un contraste explicite ou implicite avec un autre élément du discours. Pour une étude récente de la focalisation en francais vernaculaire, voir Larrivée (2020).

[5] Le consensus ne vaut que pour les constituants autres que le sujet. Quand il s'agit de structures du type (i), deux analyses différentes s'opposent : soit le groupe nominal en début de phrase est considére comme une dislocation et le pronom comme le vrai sujet de la proposition (De Cat, 2007), soit le groupe nominal est considéré comme le sujet et le pronom comme un affixe d'accord incorporé au verbe (Zribi-Hertz, 1994; Auger, 1994):

(i) Hans-Petter il est un vrai diplomate

Il est possible que les deux structures existent dans des variétés différentes ; le souci de ne pas me voir impliqué dans un débat épineux sans conséquence pour le présent article est encore une motivation pour éviter les exemples ambigus, c'est-à-dire les propositions au sujet initial. 
pas le cas en norvégien? Ce constat est d'autant plus étonnant que, dans les deux langues, la topicalisation est censée engager la périphérie gauche, voire prétendument la même position de spécifieur d'une projection universelle TopicP (voir section [2.1]).

\section{[2] THÉORIE}

Dans cette section, nous nous pencherons sur les outils théoriques nous permettant d'élucider les différences entre le français et le norvégien illustrées ci-dessus. On peut aborder cette question par deux voies, celle de la structure et celle de la dérivation. La topicalisation étant de toute évidence une opération qui affecte l'ordre des mots de la proposition de base en déplaçant un constituant en tête de phrase, une hypothèse logique serait d'attribuer les différences à des propriétés structurelles du domaine accueillant le topique. Il s'agit du domaine CP, couramment nommé la périphérie gauche, que nous considérerons dans la sous-section [2.1]. La deuxième hypothèse serait de chercher l'explication dans la dérivation, dans les étapes dont elle consiste et les propriétés syntaxiques accumulées pendant celle-ci. À cette fin j'exposerai la théorie des phases dans la section [2.2]. Il se peut fort bien que la solution fasse intervenir les deux facteurs ; c'est effectivement l'hypothèse offerte dans cette article.

\section{[2.1] La périphérie gauche}

La périphérie gauche est souvent décrite de façon simpliste comme la partie de la phrase qui se trouve à gauche du sujet, ou bien de façon plus théorique comme le domaine correspondant au CP (syntagme complémenteur) dans la grammaire générative. Selon le modèle cartographique fondé par Rizzi (1997), la périphérie gauche consiste en toute une série de projections fonctionnelles dans un ordre fixe, dont certaines associent des valeurs discursives aux constituants qu'elles accueillent. La périphérie gauche encode donc la structure informationelle de la proposition ; comme le souligne Rizzi et ses partisans, le modèle cartographique représente ainsi un élargissement du domaine de la syntaxe pour inclure des notions discursives et pragmatiques (Benincà \& Poletto, 2004; Cinque \& Rizzi, 2009; Rizzi, 2013).

Bien qu'il n'y ait pas de consensus général ni sur la structure du domaine CP ni sur le nombre exact de projections qu'il abrite, l'hypothèse de Rizzi est généralement acceptée selon laquelle le CP exprime un dualisme dans ce sens qu'il sert d'interface entre la proposition nucléaire codée par le TP et le niveau suprasyntaxique du discours. Cette nature bicéphale est codée vers le haut par la projection ForceP et vers le bas par la projection FinP, la première exprimant la force illocutoire et la deuxième certaines propriétés morphosyntaxiques de la proposition telle la finitude et peut-être le mode. Les deux projections peuvent accueillir des complémenteurs dans la position tête (Salvesen, 2014). Entre ces deux points lim- 
ites on trouve un champ de projections liées à l'expression de la structure informationelle de la proposition et qui abritent par conséquent des topiques et des focus dans la position spécifieur. La composition exacte de ce champ, le nombre de projections qui en font partie ainsi que leur localisation précise au sein du champ sont autant de questions qui occupent la recherche cartographique depuis son début. Contentons-nous de retenir une tendance (Benincà \& Poletto, 2004; Frascarelli \& Hinterhölzl, 2007), vers la reconnaissance d'une partition entre une zone relativement haute et riche abritant divers topiques et une zone plus basse et plus appauvrie abritant au moins une position de focus, ce qui nous donne la structure suivante (l'astérisque indique que la projection TopicP est potentiellement récursive) :

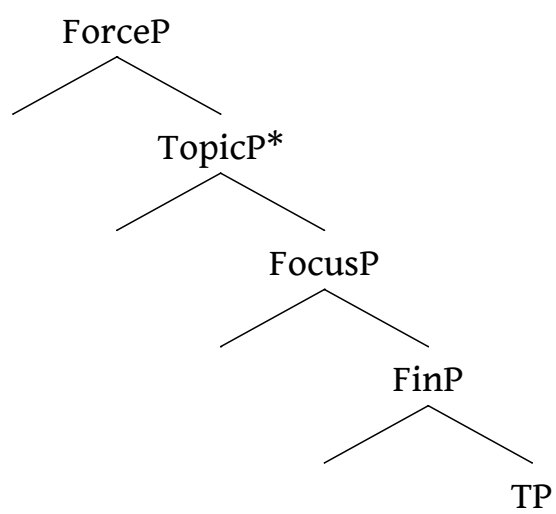

Dans le cadre de cet article, c'est donc la projection TopicP qui nous intéresse. Cependant, si l'on accepte que cette projection est, sinon universelle, du moins accessible et porteuse des mêmes traits en français et en norvégien, il est difficile de voir en quoi elle peut nous éclairer sur les différences observeés au niveau de la topicalisation dans les deux langues. L'hypothèse offerte dans cet article est que le français et le norvégien se distinguent non pas par une quelconque propriété de la projection TopicP mais par la nature de la projection FinP, à savoir son statut comme phase dans la dérivation. Avant d'entamer une analyse, il s'avère donc pertinent de considérer la théorie des phases.

\section{[2.2] La theorie des phases}

Selon la théorie des phases formulée par Chomsky (2000), la dérivation syntaxique procède par étapes, ou par phases. ${ }^{6}$ Lorsque une phase XP est complète, la tête de phase $\mathrm{X}^{0}$ transfère son complément $\mathrm{YP}$, c'est-à-dire le fragment de proposition

[6] La théorie des phases a considérablement évolué (Chomsky, 2000, 2001, 2008), et il faut admettre qu'il y a toujours peu de consensus sur les détails (Gallego, 2012). Je me permets de présenter une version simple, omettant certains points qui n'entrent pas en jeu dans le cas qui nous occupe dans cet article. Pour un exposé approfondi, voir Rouveret (2015; chapitre VIII). 
construit jusque-là, aux systèmes d'interface C-I et SM pour interprétation et articulation. Une fois transférée aux interfaces, une phase n'est plus accessible pour la computation, une hypothèse connue sous le nom de la Condition d'impénétrabilité des phases (PIC en ses sigles anglais). Il en ressort qu'un élément $Z$ destiné à entrer en relation syntaxique avec un autre élément à un moment ultérieur de la dérivation doit être évacué à une position de spécifieur à la marge de la phase ${ }^{7}$ avant le moment de transfert effectué par la tête de phase :

(6)

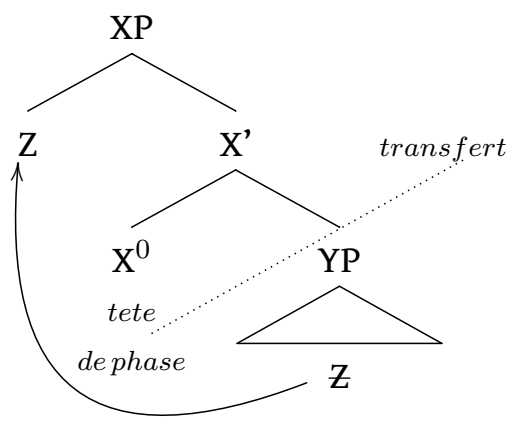

Bien que la motivation derrière la théorie des phases soit surtout d'ordre conceptuel, liée notamment au besoin de réduire la charge computationelle (Chomsky, 2000; Gallego, 2010) et d'éliminer les niveaux de représentation comme FP ou FL, son utilité sur le plan empirique est surtout celle de permettre une reformulation des effets de localité, les phases reprenant désormais le relais des barrières dans le modèle GB (Chomsky, 1986).

Reste donc de définir les domaines syntaxiques qui constituent des phases pour la computation. Le programme minimaliste étant fondamentalement guidé par les conditions de lisibilité des interfaces, on est en droit de supposer que les objets syntaxiques constituant des phases représentent des entités dotées d'une cohérence et une autonomie certaines aux niveaux sémantique et phonologique. Poursuivant cette logique minimaliste, Chomsky part de l'hypothèse que les phases sont les contreparties syntaxiques d'une proposition. Cela ne désigne pas seulement une proposition entière indépendante ou subordonnée, mais également le groupe verbal complet avec tous les rôles $\theta$ assignés. ${ }^{8}$ Fort de ce constat, Chomsky

[7] Le terme anglais est edge ; il est effectivement souhaitable d'éviter le terme périphérie, vu que la marge de la phase, qui sert de porte de sortie ('escape hatch') pour des constituants destinés à participer à une opération de Merge dans une phase ultérieur, n'a en principe rien à voir avec les domaines définis commes des périphéries dans la syntaxe ouverte et dont le rôle est surtout d'encoder la structure informationelle de la proposition. Ceci dit, la périphérie gauche de la proposition semble bien correspondre à la marge du CP, tout comme la périphérie gauche interne à la proposition, émise en hypothèse par Belletti (2004), semble correspondre à la marge du vP. Dans les deux cas, les péripheries postulées se trouvent exactement aux marges des phases $\mathrm{vP}$ et $\mathrm{CP}$ proposées par Chomsky.

[8] Ceci présuppose naturellement que le sujet en tant qu'argument externe du verbe soit assigné dans une position interne au groupe verbal (Zagona, 1982; Burton \& Grimshaw, 1992), une hypothèse désormais acceptée par de nombreux générativistes. 
considère au moins le $\mathrm{CP}$ et le vP transitif comme des phases, tandis qu'il n'accorde pas le statut phasal au TP et au VP (Chomsky, 2000: 106-107). Cette conclusion se voit renforcée au niveau phonologique dans la mesure où seuls les premiers se prêtent à des manipulations comme l'extraposition (7) et l'antéposition (8) ou bien participent dans des constructions de clivage (9) :

(7) Manger dans un bon resto parisien, tout le monde aime ça

(8) Qu'elle ait pu songer à se marier me paraissait incroyable (Aventin, Le cœur en poche, 1988, p.220 ; emprunté à Helland 2006: 125)

(9) C'est se coucher tous les soirs de bonne heure que Marcel préfère (emprunté à Rouveret 2015)

La communauté linguistique a généralement accepté la conclusion que le $\mathrm{CP}$ et le vP transitif représentent des phases, tout en proposant l'existence d'autres phases comme par exemple le PP (Abels, 2003) et le DP (Ott, 2009) : pour une discussion, voir Gallego (2009). Dans le cadre de cet article, je laisserai de côté les phases autres que le $\mathrm{CP}$, la seule à nous intéresser pour une étude de la topicalisation en français et en norvégien.

Or, après l'avènement du modèle cartographique et le $\mathrm{CP}$ éclaté (voir la section [2.1]), le CP ne désigne plus vraiment une projection, mais plutôt une abréviation pour toute une série de projections. La question se pose donc de savoir quel est le locus des têtes de phase dans la périphérie gauche mise à jour par la recherche cartographique.

Il est incontestable que, si les phases existent, la projection maximale de la proposition principale doit bien en constituer une. Il en ressort que Force ${ }^{0}$ est une tête de phase. Si nous considérons que nombre de subordonnées sont incapables d'exprimer une force illocutoire indépendante (10) et souvent même d'accueillir des topiques ou des focus dans leur périphérie gauche (11), une hypothèse logique est de considérer que Fin $^{0}$ est également une tête de phase :

(10) Sors immédiatement !

(11) *Je veux que tu sors immédiament !

(12) *si la voiture, tu me la ramènes immédiatement, je te pardonnerai

Cette conclusion semble même être présente de façon implicite chez Rizzi (1997), bien avant l'avènement de la théorie des phases, lorsqu'il affirme que la périphérie gauche sert d'interface (voir la section [2.1]) entre la proposition nucléaire et le discours. La dernière phase de la dérivation d'une proposition principale est donc introduite par la tête de phase Fin $^{0}$ et sa mission dérivationelle est d'encoder la structure informationelle par le biais des projections FocusP et TopicP. Une fois conclue cette phase, la force illocutoire est encodée par la tête 
de phase Force $^{0}$ qui transfère la proposition complète aux interfaces pour interprétation et articulation.

\section{[3] L'ANALYSE}

Avant d'entamer une analyse, répétons brièvement ce qui distingue le français et le norvégien quant au procédé de topicalisation. Le français doit forcément recourir à un pronom de reprise coréférent avec le topique déplacé dans la péripherie gauche, mais aucun changement supplémentaire ne se produit dans l'ordre des mots. Il en est tout autrement pour le norvégien, où la topicalisation déclenche automatiquement l'inversion sujet-verbe (la règle V2) sans pronom de reprise :

\section{La grammaire, Hans Petter l'enseigne depuis toujours}

(14) Grammatikk har Hans Petter undervist hele livet grammaire a Hans Petter enseigné toute vie.DEF

Pour le français, l'analyse se montre on ne peut plus simple ${ }^{9}$ lorsque tous les rôles $\theta$ ont été assignés dans le vP, l'objet direct, ayant un trait [iTop], se déplace à la marge de celui-ci pour éviter d'être transféré aux interfaces par la tête de phase $\mathrm{v}^{0}$. Dans la phase suivante, le verbe fini monte par un mouvement de tête à la position $\mathrm{T}^{0}$, attiré par un trait temporel interprétable valuant sa contrepartie ininterprétable sur le verbe lexical, lequel reçoit ainsi une morphologie précise après épelage et un ancrage temporel spécifique pour le composant interprétatif. La tête $\mathrm{T}^{0}$ est également porteuse de traits $\phi$ ininterprétables, ce qui fait de $^{0}$ une sonde ('probe') qui attire dans son spécifieur la cible la plus proche, à savoir le sujet, un groupe (pro)nominal avec des traits $\phi$ interprétables. C'est là la proposition de base française, prête pour le transfert au moment d'insertion de la tête de phase Fin ${ }^{0}{ }^{10}$ Or, l'existence d'un trait [iTop] à l'objet direct oblige ce dernier à s'évacuer à la marge de FinP avant le transfert afin d'éviter que la dérivation échoue :

[9] Que l'analyse soit simple ne veut surtout pas dire qu'elle ne pose aucune difficulté au niveau de la théorie minimaliste : se pose entre autres la question de savoir quelle est la motivation pour le mouvement du topique à la marge du vP, vu qu'il se trouve séparé de deux phases par la sonde TopicP. La sonde et la cible ne peuvent donc entrer en contact à cause de la PIC, et le mouvement pose un problème d'anticipation ('look-ahead'). Ce n'est pas le seul problème ; pour discussion, voir Rouveret 2015.

[10] Cela ne veut pas dire que la dernière phase n'entre pas en jeu, seulement qu'elle ne fait pas intervenir les têtes FocP et TopP et que, par conséquent, la dernière phase ne lexicalise rien. Cependant, toute proposition principale a une force illocutoire, et si c'est universellement la tête Force $^{0}$ qui fournit ce trait, il faut croire qu'elle est toujours activée dans la dérivation d'une proposition principale. 
(15)

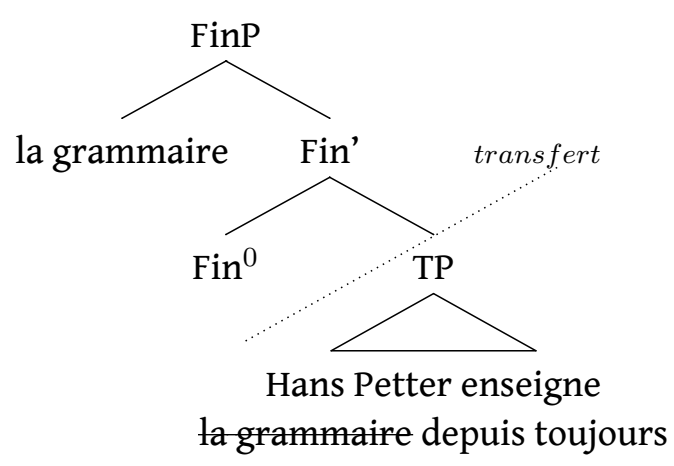

L'idée que je souhaite proposer est que le transfert effectué par la tête de phase Fin ${ }^{0}$ est à l'origine même de la périphérie gauche : celle-ci est effectivement créee par cette opération. Dans la dernière phase, achévée au moment d'insertion de la tête Force $^{0}$, la seule opération qui nous intéresse est le mouvement qui porte le topique à sa destination finale (j'omets les positions non lexicalisées comme Top ${ }^{0}$ )

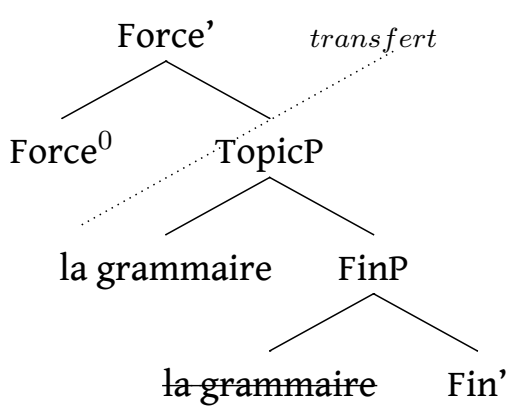

La dualité semantique du constituant topicalisé est assurée dans deux phases différentes : le rôle $\theta$ est évalué dans la première phase, celle du vP, omise dans la representation (15), et sa topicalité est assurée dans la dernière phase, en conformité avec l'idée que la structure informationelle est codée dans la périphérie gauche. Au composant phonologique, la copie basse se voit épelée comme un pronom clitique de reprise.

La nouveauté de cette analyse, à ma connaissance, est de lier la création de la périphérie gauche à l'insertion de la tête de phase $\mathrm{Fin}^{0}$; c'est le fait que cette tête intervient entre la projection TopicP et la proposition nucléaire codée par le TP qui déclenche l'emploi d'un pronom de reprise, simplement parce que tous les arguments du verbe doivent être vérifiés et exprimés au sein de la proposition nucléaire. Au-delà de cette étape dérivationelle, les constituants se trouvent en extraposition. Selon cette analyse, la périphérie gauche correspond parfaitement au domaine $\mathrm{CP}$. 
En passant maintenant au norvégien, on s'apercoit vite qu'une telle analyse ne peut convenir. S'il en était ainsi, c'est-à-dire si la topicalisation avait à franchir la même tête de phase Fin $^{0}$ pour aboutir dans la même projection dans la périphérie gauche, on s'attendrait que le norvégien procède de la même façon que le français. Tel n'est pas le cas, car il est hors de question d'employer un pronom de reprise en norvégien :
*Denne boken har Hans Petter skrevet den for studentene sine $\mathrm{Ce}$ livre a Hans Petter écrit le.ACC pour étudiants ses

Si l'on se permet une introspection sur la nature de l'agrammaticalité de (17), le sentiment est celui d'une forte redondance, voire une incohérence : on ne sait pas quoi faire avec le pronom de reprise. Chose intéressante, les faits sont les mêmes pour toutes les langues V2 germaniques. Ce constat nous fournit une indication utile sur la dérivation. Il est généralement admis que le verbe fini dans les langues V2 doit se déplacer dans une position plus haute que celle occupée par le verbe dans les langues non V2. Selon l'analyse traditionnelle du phénomène V2, qui remonte au travail important de den Besten (1983), cette position se trouve dans la tête de la projection CP. Cette hypothèse a connu un grand succès et fait aujourd'hui, peu ou prou, l'unanimité des chercheurs travaillant sur la syntaxes des langues germaniques (Holmberg 1986; Vikner 1995; pour un aperçu, voir KlævikPettersen 2019, chapitre 2); mais toujours est-il que cette analyse a besoin d'une mise à jour après l'avènement du CP éclaté, car le concept de $\mathrm{CP}$ n'est plus suffisamment précis. La question se pose donc de savoir dans quelle position dans le domaine $\mathrm{CP}$ le verbe fini atterrit dans les langues V2.

$\mathrm{Vu}$ que le seul constituant normalement admis dans la position directement devant le verbe fini peut être soit un focus, soit un topique, on est en droit de supposer que cette position est la tête Fin ${ }^{0}$. Le fait qu'une proposition V2 avec inversion sujet-verbe soit possible même dans certains types de subordonnée (18) renforce cette hypothèse, car il faut croire que le complémenteur réside dans la position Force ${ }^{0}$ dans ces cas : ${ }^{11}$
Hans Petter sa
at lingvistikk hadde han alltid
elsket Hans Petter dire.PERF que linguistique avait il toujours aimé 'Hans Petter a dit que la linguistique, il l'avait toujours aimée'

[11] Le relecteur demande s'il existe des preuves indépendantes d'une position haute de complémenteur dans la périphérie gauche en norvégien. À ma connaissance, il est très difficile de trouver des preuves morphosyntaxiques directes de cette position dans les langues scandinaves en général ; la construction à double complémenteur ('complementiser doubling'), répandue dans plusieurs langues romanes, n'existe par exemple pas en norvégien. Quoi qu'il en soit, il est difficile de voir comment on peut éviter la conclusion qu'un exemple comme (18) implique la projection ForceP, car l'inversion sujet-verbe montre que le verbe s'est deplacé dans le domaine $\mathrm{C}$, ce qui veut dire que le complémenteur doit se trouver dans une position supérieure à TopicP. 
L'analyse que je souhaite proposer est donc la suivante : la dérivation procède de façon identique pour la phase vP. Une fois construit le TP, la tête Fin ${ }^{0}$ est insérée dans la structure. C'est à ce moment-là qu'intervient la particularité des langues V2, car cette tête porte un trait ininterprétable qui a pour effet d'attirer le verbe fini de sa position dans $\mathrm{T}^{0}$. Ce faisant, le verbe rend inactive la phase, ce qui veut dire qu'aucune périphérie gauche n'est créee. Par conséquent, la proposition nucléaire se rallonge pour inclure non seulement le spécifieur de FinP, mais également la projection TopicP, car la phase ne s'achève qu'avec l'insertion de la tête de phase Force $^{0}$ :

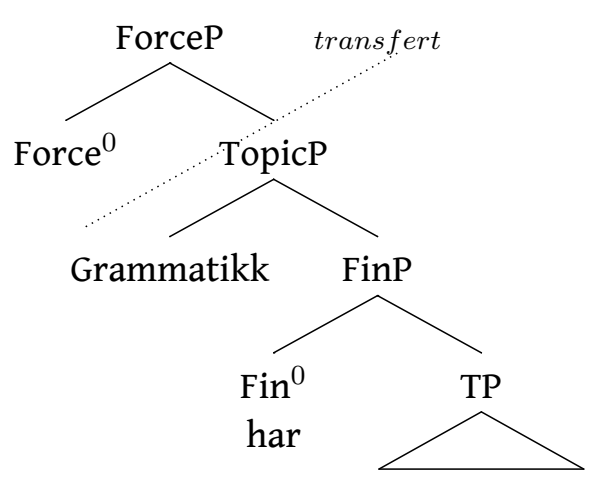

Hans Petter har undervist grammatikk hele livet

L'idée que la phase peut être desactivée par le mouvement du verbe n'est pas une stipulation sans motivation théorique et conceptuelle. Retenons d'abord que le verbe est un constituant particulier dans la mesure où la proposition entière en représente une projection. Autant dire que si le verbe monte, la proposition fait de même. C'est sûrement pour cette raison que le verbe fini, à la différence d'un $\mathrm{vP}$, ne peut aboutir dans la périphérie gauche; il ne peut par exemple jamais être topicalisé :

(20) *Lis le livre de grammaire de Hans Petter, je (fais) souvent

Dans le cadre de la théorie des phases, il y a cependant une raison concrète qui confère une probabilité à cette hypothèse, à savoir l'idée développée dans Chomsky (2008) selon laquelle les phases sont définies par la présence de traits inintérprétables sur les têtes de phase ; une fois évalués, ces traits sont aussitôt éliminés par l'opération de transfert qui soumet la phase à l'inspection des interfaces. Dans les langues V2 comme le norvégien, la tête Fin ${ }^{0}$ est de toute évidence porteuse d'un trait attirant le verbe, et vu que ce trait n'a aucune répercussion sur l'interprétation, il faut croire qu'il s'agit justement d'un trait ininterprétable. La conclusion se présente d'elle-même : la tête $\mathrm{Fin}^{0}$, qui a pleinement le statut phasal dans les langues non V2 comme le français et qui se trouve, selon l'hypothèse 
présentée ici, à l'origine même de la création de la périphérie gauche, ne constitue pas une tête de phase dans les langues V2.

Voilà qui explique le lien entre le mouvement du verbe et le type de topicalisation: en français, la topicalisation est une opération qui franchit la phase séparant la proposition nucléaire et la périphérie gauche, et l'emploi d'un pronom de reprise découle de façon naturelle de ce constat. En français, et probablement dans les langues non V2 en général, les termes dislocation, périphérie gauche et domaine $C P$ sont pleinement équivalents. En norvégien et, par hypothèse, dans les langues V2 en géneral, la topicalisation a lieu à l'intérieur de la proposition nucléaire sans jamais engager la périphérie gauche, car le mouvement du verbe à la tête Fin $^{0} \mathrm{a}$ désactivé le statut de celle-ci comme tête de phase. ${ }^{12}$ Il va sans dire qu'un pronom de reprise produit, non seulement un pléonasme, mais une nette incohérence au niveau de la structure argumentale du verbe. Autrement dit, dans les langues V2 le domaine $\mathrm{CP}$ ne correspond pas à la périphérie gauche, et les topiques ne sont pas disloqués.

Faut-il admettre que les langues V2 n'ont pas de périphérie gauche? Ce serait oublier que le domaine $\mathrm{CP}$ comprend encore une tête de phase, à savoir la tête Force ${ }^{0}$. C'est probablement cette tête qui entre en jeu dans les cas, très restreints, où le verbe peut se voir relégué à la troisième position de la proposition, une possibilité seulement admise si la proposition contient, précisement, un pronom de reprise (21). Le constituant initial est clairement disloqué, et le fait que la proposition nucléaire suivante contienne une inversion sujet-verbe indique que le verbe se trouve dans Fin $^{0}$. Le pronom de reprise occupe probablement la position dans le spécifieur de TopicP, ce qui mène à la conclusion que le constituant initial a franchi la tête de phase Force $^{0}$, peut-être pour atterir dans son spécifieur ${ }^{13}$ :
Hans Petter, han har alle studentene sansen for Hans Petter lui ont tous étudiants.DEF préférence pour 'Hans Petter, tous les étudiants l'apprécient'

[12] Le relecteur pose la question de savoir si la périphérie gauche disparaît complètement en norvégien, ou si elle est seulement interne à la proposition. En effet, la réponse est bien que la périphérie gauche disparaît, mais il faut souligner que cela n'est qu'une question de terminologie, car la périphérie gauche est prise dans un sens relatif et non pas absolu. Le domaine $C$ et les projections qu'il contient ne peuvent en aucun cas disparaître, mais certaines de ces projections peuvent se trouver à l'intérieur de la proposition nucléaire. Concrètement, la périphérie gauche désigne ici les projections situées au-dessus de la première tête de phase du domaine $C$. En français, cette tête est $\operatorname{Fin}^{0}$ et la périphérie gauche commence par conséquent après le transfert effectué par Fin ${ }^{0}$. En norvégien, selon la présente analyse, Fin ${ }^{0}$ n'est pas une tête de phase et aucun transfert n'a lieu, ce qui veut dire qu'aucune périphérie n'est créée à ce point de la dérivation.

[13] Une autre possibilité serait qu'il existe des projections au-dessus de ForceP, comme par exemple le FrameP postulé par certains chercheurs (Benincà \& Poletto, 2004; Haegeman \& Greco, 2018) et dont le rôle est d'accueillir un certain type de topique. Il est également possible que les éléments se trouvant dans cette projection soit générés directement dans la périphérie gauche. Cela n’a aucun impact pour l'hypothèse présentée ici selon laquelle le pronom de reprise s'avère possible et obligatoire seulement s'il se trouvé séparé de son expression coréférentielle par une phase. 


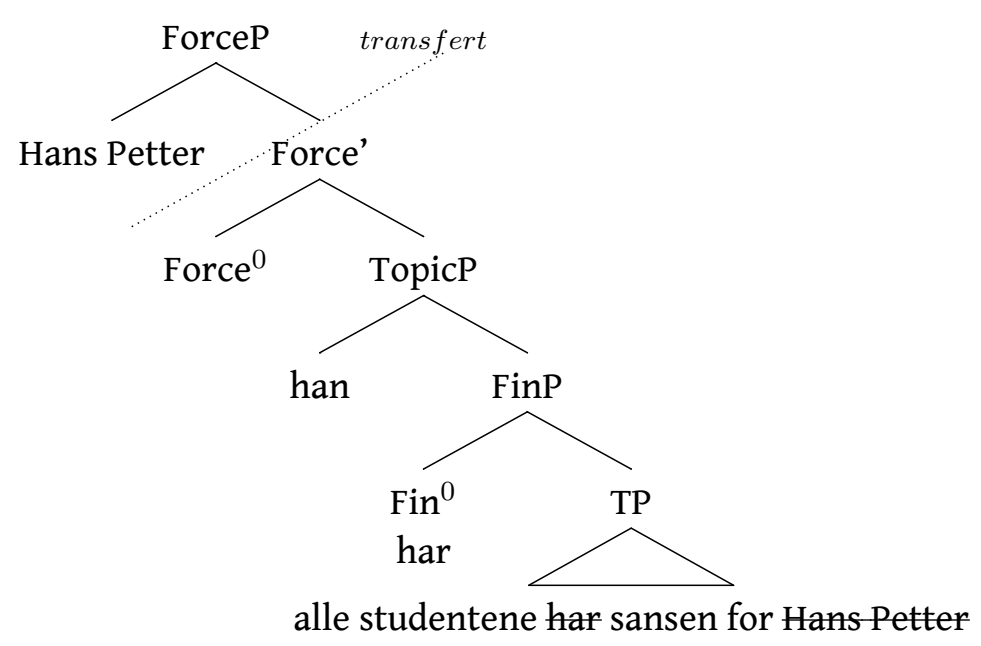

Même dans le sens restreint du terme proposé ici, les langues V2 semblent donc bien être munis d'une périphérie gauche, c'est-à-dire d'un domaine de dislocation extérieur à la proposition nucléaire. Cependant, l'utilité d'une telle dislocation se voit quelque peu diminuée par le fait que la topicalisation peut être réalisée à l'interieur de la proposition même par une simple structure V2. De fait, il est difficile de voir en quoi se distinguent une proposition V3 avec reprise telle (21) et une topicalisation V2 invertée sans reprise.

\section{[3.1] L'ancien français}

Avant de clore cette section, je souhaite faire remarquer un point important qui renforce l'hypothèse tout juste présentée. Bien sûr, il est possible que cette analyse soit fautive et que la corrélation entre topicalisation et dislocation avec reprise soit dûe au hasard, qu'il s'agisse simplement d'une construction partagée par les langues romanes à cause de leur origine commune et leur développement parallèle, tout comme la corrélation entre topicalisation sans reprise et V2 dans les langues germaniques pourrait être un fait accidentel pour les même raisons.

C'est là que l'ancien français nous aide à voir plus clair. Au $13^{\text {ème }}$ siècle, le français était une langue V2 selon la vaste majorité des chercheurs qui se sont penchés sur cette question (Vanelli et al. 1985; Roberts 1993; Vance 1997; Rouveret 2004; Labelle 2007; Salvesen 2013; Steiner 2014; Klævik-Pettersen 2019; mais voir Kaiser 2002; Elsig 2012 pour une conclusion différente). Ici encore, la corrélation entre V2 et topicalisation sans reprise est manifeste dans tous les documents en prose de cette période, comme l'attestent les exemples suivants :

(23) Tel don te fais je, biaus ami...(Le Roman de Tristan en Prose (Curtis, 1963: 40))

(24) Car cest don li dona Nostre Sires...(La Vie de Saint Eustace, XXXIX (Murray, 1929: 45) 
Il est également possible de trouver des données qui attestent d'une périphérie haute qui mène à des structures V3 avec, on s'y attendrait maintenant, un pronom de reprise (25). La seule différence avec les séquences V3 dans les langues germaniques modernes est que le pronom de reprise ne peut occuper la position devant le verbe en ancien français. Ce constat s'explique par le simple fait que les pronoms étaient déjà des clitiques en ancien français et qu'à la différence de la langue moderne, un pronom clitique ne peut ouvrir la proposition selon la loi Tobler-Mussafia :

(25) Tote la terre que li barbarin avoient saisie, il la delivra...(La Vie de Saint Eustace, XXXVII (Murray, 1929: 30)

En conclusion, il semble bien que la distinction pertinente, en ce qui concerne le procédé de topicalisation, soit celle entre les langues V2 et les langues non V2, et non pas entre les langues germaniques et les langues romanes.

\section{CONCLUSION}

Sans présenter de nouvelles données, cet article a proposé une analyse nouvelle de la topicalisation en français et en norvégien qui fait intervenir le concept de dérivation par phase. L'idée centrale est que le mouvement du verbe a des répercussions importantes sur la topicalisation; si le verbe se déplace à la tête de phase Fin $^{0}$, comme c'est exceptionellement le cas dans les langues V2, ce mouvement parvient à désactiver la phase, avec la conséquence que la proposition nucléaire se rallonge dans le domaine $\mathrm{CP}$ pour inclure également la projection TopicP. J'ai proposé que cela peut expliquer pourquoi les langues V2 n'ont pas recours à un pronom de reprise : le topique et le pronom de reprise se trouveraient tous les deux à l'intérieur de la proposition nucléaire et il en résulterait une incohérence au niveau de la structure argumentale. Il en est autrement pour le français moderne comme langue non V2, car la topicalisation franchit une phase et aboutit dans la périphérie gauche créee par Fin ${ }^{0}$.

Il va sans dire que cet article ne constitue qu'une première ébauche vers une nouvelle théorie de la syntaxe propositionelle. Plusieurs questions importantes restent à élucider, comme par exemple celle de savoir en quoi cette théorie peut expliquer la focalisation, où un constituant est prétendument aussi attiré dans une position dans le domaine $\mathrm{C}$, mais où ni le français ni le norvégien n'emploie un pronom de reprise. Ceci dit, j'estime que l'analyse présentée ici peut nous permettre de voir sous un nouveau jour la particularité syntaxique des langues V2, et qu'elle peut suggérer une autre manière, sur le plan diachronique cette fois, de contempler la nature du processus, mal compris, qui mène à la création d'une langue V2. Dans l'optique de cet article, il s'agirait, faute de terme meilleur, d'une grammaticalisation de la périphérie gauche effectuée par le mouvement du verbe 
au domaine $\mathrm{CP}$.

\section{REMERCIEMENTS}

Je tiens à remercier les éditeurs pour cette occasion d'offrir mes hommages à Hans Petter. Son livre de grammaire a été comme un texte sacré (et parfois un sacré texte) pour moi pendant mes études et j'y reviens régulièrement pour vérifier quelque détail de cette langue complexe qu'est le français. Merci, Hans-Petter, pour nos discussions toujours intéressantes et pour tous les bons conseils. Joyeux anniversaire!

\section{REFERENCES}

Abels, Klaus. 2003. Successive cyclicity, anti-locality, and adposition stranding. University of Connecticut PhD dissertation.

Auger, Julie. 1994. Pronominal Clitics in Québec Colloquial French: A Morphological Analysis. University of Pennsylvania PhD dissertation.

Belletti, Adriana. 2004. Aspects of the Low IP Area. In Luigi Rizzi (ed.), The Structure of CP and IP: The Cartography of Syntactic Structures, vol. 2, 16-51. Oxford and New York: Oxford University Press.

Benincà, Paola \& Cecilia Poletto. 2004. Topic, Focus, and V2: Defining the CP Sublayers. In Luigi Rizzi (ed.), The Structure of CP and IP Oxford Studies in Comparative Syntax, Oxford and New York: OUP.

den Besten, Hans. 1983. On the interaction of root transformations and lexical deletive rules. In Werner Abraham (ed.), On the Formal Syntax of the Westgermania (Linguistik Aktuell 3), 47-132. Amsterdam: John Benjamins.

Blasco-Dulbecco, Mylène. 1999. Les dislocations en français contemporain: étude syntaxique. Paris: H. Champion.

Burton, Strang \& Jane Grimshaw. 1992. Coordination and VP-Internal Subjects. Linguistic Inquiry 305-313.

Chomsky, Noam. 1986. Barriers. Cambridge, MA: MIT Press.

Chomsky, Noam. 2000. Minimalist Inquiries. In Roger Martin, Michaels David \& Juan Uriagereka (eds.), Step by Step: Essays in Minimalist Syntax in Honor of Howard Lasnik, 89-155. Cambridge, MA: MIT Press.

Chomsky, Noam. 2001. Derivation by phase. In Michael Kenstowicz (ed.), Ken Hale: A life in language, 1-52. Cambridge, MA: MIT Press. 
Chomsky, Noam. 2008. On Phases. In C. P. Otero R. Freidin \& M.-L. Zubizarreta (eds.), Foundational Issues in Linguistic Theory, 133-166. MIT Press.

Cinque, Guglielmo \& Luigi Rizzi. 2009. The Cartography of Syntactic Structures. In Bernd Heine \& Heiki Harrog (eds.), The Oxford Handbook of Linguistic Analysis, 51-65. Oxford: OUP.

Curtis, Renée L. (ed.). 1963. Le Roman de Tristan en Prose. Tome 1. München: Max Hueber Verlag.

De Cat, Cécile. 2007. French Dislocation. Interpretation, Syntax, Acquisition. New York: Oxford University Press.

Elsig, Martin. 2012. Subject-verb inversion in 13th century German and French. In Kurt Braunmüller \& Christoph Gabriel (eds.), Multilingual Individuals and Multilingual Societies (Hamburg Studies on Multilingualism 13), 223-239. Amsterdam: John Benjamins.

Frascarelli, Mara \& Roland Hinterhölzl. 2007. Types of Topics in German and Italian. In Kerstin Schwabe \& Susanne Winkler (eds.), On information structure, meaning and form: generalizations across languages, 87-116. Amsterdam: John Benjamins.

Gallego, Angel J. 2010. Phase Theory. Amsterdam; Philadelphia: John Benjamins.

Gallego, Ángel J. 2009. Phases and Variation: Exploring the Second Factor of Language. In J. van Craenenbrock (ed.), Alternatives to Cartography, 109-152. Mouton de Gruyter.

Gallego, Ángel J. 2012. Introduction : A framework of phases for linguistic theory. In Ángel J. Gallego (ed.), Phases, Mouton de Gruyter.

Haegeman, Lilian \& Ciro Greco. 2018. West Flemish V3 and the interaction of syntax and discourse. Journal of Comparative Germanic Linguistics 21. 1-56.

Helland, Hans Petter. 2006. Ny fransk grammatikk. Universitetsforlaget.

Helland, Hans Petter, Christine Nilsen \& Terje Lohndal. 2020. La topicalisation en français et norvégien. Scolia 34.

Holmberg, Anders. 1986. Word order and syntactic features in the Scandinavian languages and English. University of Stockholm PhD dissertation.

Kaiser, Georg A. 2002. Verbstellung und Verbstellungswandel in den romanischen Sprachen (Linguistische Arbeiten 465). Tübingen: Max Niemeyer. 
Klævik-Pettersen, Espen. 2019. Inversion, V-to-C, and verb second: an investigation into the syntax and word order of Old French and Late Latin. University of Oslo PhD dissertation.

Labelle, Marie. 2007. Clausal Architecture in Early Old French. Lingua 117(1). 289316.

Larrivée, Pierre. 2020. Le focus initial en français vernaculaire. Scolia 34. 33-50.

Murray, Jessie (ed.). 1929. La Vie de Saint Eustace. Paris: Librairie Ancienne Honoré Champion.

Ott, Dennis. 2009. The conceptual necessity of phases : some remarks on the minimalist enterprise. In K. Grohmann (ed.), Explorations of phase theory: Interpretation at the interfaces, 253-275. Mouton de Gruyter.

Rizzi, Luigi. 1997. The fine structure of the left periphery. In Liliane Haegeman (ed.), Elements of Grammar: Handbook of Generative Syntax (Kluwer International Handbooks of Linguistics 1), 281-337. Dordrecht: Kluwer.

Rizzi, Luigi. 2013. Notes on cartography and further explanation. International Journal of Latin and Romance Linguistics 25(1). 197-226.

Roberts, Ian. 1993. Verbs and Diachronic Syntax: A Comparative History of English and French (Studies in Natural Language and Linguistic Theory 28). Dordrecht: Kluwer.

Rouveret, Alain. 2004. Les clitiques pronominaux et la périphérie gauche en ancien français. Bulletin de la Société de linguistique de Paris 99(1). 181-237.

Rouveret, Alain. 2015. Arguments minimalistes. ENS Éditions. doi:10.4000/books. enseditions.5588.

Salvesen, Christine Meklenborg. 2013. Topics and the Left Periphery: A Comparison of Old French and Modern Germanic. In Terje Lohndal (ed.), In Search of Universal Grammar: From Old Norse to Zoque, 131-172. Amsterdam: John Benjamins.

Salvesen, Christine Meklenborg. 2014. Le complémenteur que et la périphérie gauche : analyse diachronique. Syntaxe et Sémantique 1(15). 47-80.

Steiner, B. D. 2014. The evolution of information structure and verb second in the history of French. Indiana University $\mathrm{PhD}$ dissertation.

Vance, Barbara S. 1997. Syntactic Change in Medieval French: Verb-Second and Null Subjects (Studies in Natural Language and Linguistic Theory 41). Dordrecht: Kluwer. 
Vanelli, Laura, Lorenzo Renzi \& Paola Benincà . 1985. Typologie des pronoms sujets dans les langues romanes. In Actes du XVIIème Congrès International de Linguistique et Philologie Romanes (Aix-en Provence, 29 août - 3 septembre 1983), vol. 3, 163-176. Aix-en Provence: Université de Provence.

Vikner, Stein. 1995. Verb Movement and Expletive Subjects in the Germanic Languages. Oxford: OUP.

Zagona, Karen. 1982. Government and proper government of verbal projections. University of Washington $\mathrm{PhD}$ dissertation.

Zribi-Hertz, Anne. 1994. The syntax of nominative clitics in standard advanced French. In Cinque et al. (ed.), Paths Towards Universal Grammar. Studies in Honor of Richard S. Kayne, 453-472. Washington DC: Georgetown University Press. 